\title{
Clustering and superspreading potential of severe acute respiratory syndrome coronavirus 2 (SARS- CoV-2) infections in Hong Kong
}

\section{Dillon Adam}

The University of Hong Kong

Peng Wu ( $\sim$ pengwu@hku.hk)

The University of Hong Kong https://orcid.org/0000-0003-1157-9401

Jessica Wong

The University of Hong Kong

\section{Eric Lau}

The University of Hong Kong

\section{Tim Tsang}

The University of Hong Kong

\section{Simon Cauchemez}

Pasteur Institut

\section{Gabriel Leung}

The University of Hong Kong https://orcid.org/0000-0002-2503-6283

\section{Benjamin Cowling}

The University of Hong Kong https://orcid.org/0000-0002-6297-7154

\section{Research Article}

Keywords: coronavirus, SARS-CoV-2, COVID-19, superspreading, transmission, public health

Posted Date: May 21st, 2020

DOI: https://doi.org/10.21203/rs.3.rs-29548/v1

License: (c) (1) This work is licensed under a Creative Commons Attribution 4.0 International License. Read Full License

Version of Record: A version of this preprint was published on September 17th, 2020. See the published version at https://doi.org/10.1038/s41591-020-1092-0. 
Clustering and superspreading potential of severe acute respiratory syndrome coronavirus 2 (SARS-CoV-2) infections in Hong Kong

Dillon C. Adam ${ }^{1,2}$, Peng Wu ${ }^{1}$, Jessica Y. Wong ${ }^{1}$, Eric H. Y. Lau ${ }^{1}$, Tim K. Tsang ${ }^{1}$, Simon Cauchemez $^{3}$, Gabriel M. Leung ${ }^{1 *}$, Benjamin J. Cowling ${ }^{1 *}$

*Joint senior authors

\section{Affiliations:}

1. WHO Collaborating Centre for Infectious Disease Epidemiology and Control, School of Public Health, Li Ka Shing Faculty of Medicine, The University of Hong Kong, Hong Kong Special Administrative Region, China.

2. Biosecurity Program, Kirby Institute, University of New South Wales, Sydney, Australia.

3. Mathematical Modelling of Infectious Diseases Unit, Institut Pasteur, Paris, France.

\section{Corresponding author:}

Peng Wu, School of Public Health, Li Ka Shing Faculty of Medicine, The University of Hong Kong, 7 Sassoon Road, Pokfulam, Hong Kong

Tel: +852 3917 6746; Email: pengwu@hku.hk 
23 Word count (abstract): 138

24 Word count (main text, excluding methods): 2,861

25

26 Key words: coronavirus; COVID-19; superspreading; transmission; public health 
Superspreading events have characterised previous epidemics of severe acute respiratory syndrome coronavirus (SARS-CoV) and Middle East respiratory syndrome coronavirus (MERS-CoV) infections. Using contact tracing data, we identified and characterized SARS-CoV-2 clusters in Hong Kong. Given a superspreading threshold of 6-8 secondary cases, we identified 5-7 probable superspreading events and evidence of substantial overdispersion in transmissibility, and estimated that $20 \%$ of cases were responsible for $80 \%$ of local transmission. Among terminal cluster cases, 27\% (45/167) ended in quarantine. Social exposures produced a greater number of secondary cases compared to family or work exposures $(\mathrm{p}<0.001)$ while delays between symptom onset and isolation did not reliably predict the number of individual secondary cases or resulting cluster sizes. Public health authorities should focus on rapid tracing and quarantine of contacts, along with physical distancing to prevent superspreading events in high-risk social environments.

Severe acute respiratory syndrome coronavirus 2 (SARS-CoV-2) is a novel betacoronavirus that was first identified in patients in China in December 2019 and January 2020, and has since caused over four million confirmed human infections worldwide and more than 300,0000 deaths (1). Disease caused by SARS-CoV-2 is termed coronavirus disease 2019 (COVID-19) and was declared a global pandemic on March 11, 2020 (2). Epidemics of severe acute respiratory syndrome coronavirus (SARS-CoV) in 2002-03 and Middle-East respiratory syndrome coronavirus (MERS- 
$\mathrm{CoV}$ ) since 2013 have been notable for the occurrence of superspreading events (SSEs) (3-6). SSEs are generally defined as outbreaks in which a small number of cases infect a large number of secondary cases well-above the expected average (7). For SARS-CoV, with an basic reproductive number $\left(\mathrm{R}_{0}\right)$ of around 3, (8), greater than eight or 10 secondary cases have been suggested to constitute SSEs $(9,10)$, while for MERS-CoV, SSEs have reportedly involved up to 82 secondary cases (11). For SARS$\mathrm{CoV}-2$, the Ro has been estimated between 2 and $3(12,13)$, and the degree to which SSEs are involved in transmission of COVID-19 remains unclear. Here, we used contact tracing data to identify and describe clusters of SARS-CoV-2 infection in Hong Kong and estimate the degree of variance or overdispersion in transmissibility, and therefore the potential for SARS-CoV-2 SSEs.

\section{RESULTS}

Cases and Clusters of SARS-CoV-2 in Hong Kong

As of 28 April 2020, there have been a total of 1,037 laboratory-confirmed cases of SARS-CoV-2 infection in Hong Kong, and one probable case based on clinical and epidemiological features. The first case of SARS-CoV-2 infection in Hong Kong was confirmed on January 23, 2020, imported from Hubei, China (Onset January 18, 2020. The first local cases without known travel history were confirmed on February 4, 2020 among a family cluster with symptom onsets ranging from January 22, 2020 to February 4, 2020 indicating potentially undocumented community transmission had occurred in mid-January 2020 prior to the first travel restrictions implemented from 
January 25, 2020 (Figure 1). Stable numbers of sporadic and cluster cases were detected until early March when a substantial increase in the number of imported cases was observed (Figure 1). Cases continued to rise until a total ban on nonresident entry and mandatory 14-day quarantine for all arrivals was implemented. A rise in the number of local cases including clusters initiated by local or imported cases saw the implementation of various physical distancing measures such as restrictions on gatherings and bar closures to prevent community transmission (Figure 1).

Overall the majority $(51.9 \% ; 539 / 1,038)$ of SARS-CoV-2 infections in Hong Kong have been associated with at least one of 135 known clusters. The median cluster size was two and the largest involved 106 local cases. The remaining 38.9\% (210/539) of cluster cases solely involved imported cases where no onward local transmission could be identified. Most clusters comprised only imported cases (82/135; 60.7\%), followed by clusters initiated by an imported case $(30 / 135 ; 22.2 \%)$ and clusters initiated by a local case $(23 / 135 ; 17.0 \%)$. Among the 499 sporadic cases not linked to any cluster, $90.0 \%$ of infections were acquired overseas (449/499), while the remaining $50(10.0 \%)$ had no history of travel and could not be linked to any other case either as a source or subsequent generation. Of cluster cases, 225 (41.7\%; 225/539) belonged to clusters initiated by another local case, compared to $19.3 \%$ (104/539) of cluster cases which belonged to clusters initiated by an imported case. The composition of clusters is described further in Supplementary Table 1. 
Transmission Pairs E Individual variation in SARS-CoV-2 transmission

From the 539 infections that occurred in clusters, all 210 solely imported cases $(210 / 539 ; 38.9 \%)$ were excluded from subsequent analysis due to uncertainties concerning transmission within each cluster whilst overseas. Within the remaining 53 clusters initiated by a local or imported infection, $245(245 / 329 ; 74.5 \%)$ could be linked resulting in 171 unique infector-infectee transmission pairs with 94 unique infectors. The age of distribution of infectors and infectees was not significantly different (Supplementary Figure 1A) however a positive trend by age can be seen in the transmission pair matrix (Supplementary Figure 1B).

Figure 2A shows the empirical serial distribution between all infector-infectee pairs and fitted normal and lognormal distributions. The median serial interval was 4 days (IQR 3 - 8 days), and the mean of the fitted normal distribution was 5.6 days (standard deviation 4.3 days). We observed at least eight instances of likely presymptomatic transmission where symptom onset of the infectee preceded that of the infector by one day $(\mathrm{N}=2)$ or occurred on the same day $(\mathrm{N}=6)$. Thirty-four unique infectors $(35 / 94 ; 37.2 \%)$ were linked to two or more secondary cases, and the largest number of individual secondary cases was 11. From the empirical offspring distribution and fitted negative binomial distribution shown in Figure 2B, we estimated an observed reproductive number $(\mathrm{R})$ of 0.58 (95\% CI: $0.45-0.71)$ and dispersion parameter $(k)$ of 0.45 (95\% CI: $0.31-0.76)$. Given a superspreading 
threshold of 6-8 secondary cases, we directly observed 2-4 SSEs where the source was known. Likelihood analysis based on final cluster sizes increased the estimate of R to 0.75 (95\% CI: $0.6-0.96)$ and decreased estimates of $k$ to 0.37 (95\% CI: $0.16-$ 1.16). Based on these estimates we determined a coefficient of variation of 2.5 and inferred that approximately $20 \%$ of SARS-CoV-2 infections are responsible for $80 \%$ of all transmission events in Hong Kong (Table 1). Epoch analyses by wave (before or after March 1, 2020) showed similar estimates of $\mathrm{R}$ and $k$ (Supplementary Table 2) from their respective offspring distributions (Supplementary Figure 2). A peak however in the number of index cases per day could be seen in wave two (Supplementary Figure 3). Parameter estimates and distributions for all fitted distributions are shown in Supplementary Tables 3 \& 4, and Supplementary Figures $4 \& 5$.

\section{Chains of SARS-CoV-2 transmission in Hong Kong}

The largest local cluster was 106 cases and was traced back to multiple social exposures among a collection of bars across Hong Kong (Figure 3A). Evidence suggested this "bar and band" cluster originated in Lan Kwai Fong among a few staff and customers before being spread to additional venues by a number of musicians, however the source and chains of transmission between many bar cases could not be determined from epidemiological data. The earliest reported exposure was reported on March $7^{\text {th }}$ among two customers who later presented with symptoms on March $11^{\text {th }}$ (Supplementary Figure 6A). The earliest symptom onset 
was among two staff members on March $10^{\text {th }} \& 11^{\text {th }}$, however neither case was confirmed until March $24^{\text {th }}$ and $25^{\text {th }}$ and neither reported their history of exposure at the bar during this time. Given the serial intervals between these four cases and later cases (Supplementary Figure 6A), it is possible that an undetected source may have initially infected these staff and customers on March 7, before being spread to the musicians. The earliest onset among the musicians was on March 17, with most subsequently affected customers reporting exposures between March 17 and March 20 (Supplementary Figure 6C), which is suggestive of at least one or more probably SSEs within this exposure period. The bars were voluntarily closed from March 23 for cleaning prior to mandated closures from April 3. Of the 73 primary bar cases, 39 customers, 20 staff, and 14 musicians were infected, while the remaining 33 infections were secondary, tertiary or quaternary contacts (Supplementary Figure 6A). This single outbreak accounts for $10.2 \%(106 / 1,038)$ of all cases in Hong Kong regardless of the source, but $30.4 \%$ of all local cases acquired in Hong Kong (106/349). Evidence of asymptomatic transmission was determined in a single case (a staff member at the bar) who was placed in quarantine before their wife subsequently presented with symptoms and tested positive. Supplementary Figure $6 \mathrm{~B}$ shows the age distribution of all cases associated with this cluster.

Figure 3B describes a cluster of 21 cases linked to a wedding. Ten cases resulted from a previous social exposure (SSE), of which four cases subsequently attended the wedding. Individual transmission pairs within the wedding could not be 
determined, however there were at least seven secondary infections, and therefore another potential SSE, and an additional two tertiary cases among family members of the wedding guests. A final potential SSE of undetermined origin was associated with religious activities at a local temple and resulted in 11 primary cases and 18 recorded cases total (Figure 3C). Cases reported multiple exposures over a number of days such that a single point source exposure was unlikely. Six secondary cases were linked via family exposures. The last case who worked at the temple was also infected however remained asymptomatic. It is unknown if this case was the source of the temple exposures or was infected by the undetermined source/s.

Figure 3D shows all other local cluster cases and chains of SARS-CoV-2 transmission in Hong Kong. In total, 51 subsequent cases were quarantined. Among terminal transmission cases, $26.9 \%$ (45/167) ended in quarantine (excluding five quarantined bars cases whose terminal transmission position was ambiguous; Figure 4A; Supplementary Table 5). The odds that a terminal case ended in quarantine was 16.97 (95\% CI: 2.27 - 126.69), while the probability that a case was terminal and quarantined was $21.0 \%(45 / 214)$ compared to $57.0 \%$ (122/214) for cases who were terminal but not quarantined (Supplementary Table 5). Transmission within families was the most frequent event among all recognised transmission pairs $(96 / 171 ; 56.1 \%)$ followed by transmission in social settings $(55 / 171 ; 32.2 \%)$ and at work $(20 / 171$; $11.1 \%)$. However, there was very strong evidence that the number of individual secondary cases resulting from social exposures was significantly higher compared 
to work or family exposures $(\mu=2.89$ vs. $1.36 ; \mathrm{p}<0.001$, negative binomial regression model).

Relationship between delayed case isolation and potential COVID-19 cluster size

Figure 4A shows the median individual delay from symptom onset-toconfirmation/isolation among SARS-CoV-2 infections in Hong Kong by cluster membership on the basis of size. We found that the delay from symptom onset to confirmation among cluster cases did not predict the overall cluster size although if excluding the two largest clusters there was a slight increase in onset-to-isolation delays in some of the larger clusters (Figure 4A). Delay from symptom-onset to isolation among infectors similarly was not associated with the number of secondary cases or the overall size of each cluster $(p=0.955$, linear regression; Figure 4B).

\section{DISCUSSION}

Public health measures have successfully suppressed transmission of SARS-CoV-2 infections in Hong Kong with an estimated reproductive number below 1 (Table 1; $\mathrm{R}=0.58,95 \%: 0.45-0.71$ ) compared to estimates of the basic reproductive number $\mathrm{R}_{0}$ of 2-3 $(12,13)$, and our estimate varied only marginally between phase one (Jan-Feb) and phase two of the epidemic in Hong Kong (Mar-Apr) (Supplementary Table 2; $\mathrm{R}=0.60,95 \%: 0.35-0.94$ and $\mathrm{R}=0.56,95 \%: 0.43-0.70)$. Thirty-three percent $(349 / 1,038)$ of all SARS-CoV-2 infections in have been acquired within Hong Kong, either within clusters or as sporadic local cases occurring through limited community 
transmission that has been controlled through a range of public health measures (14). Our results indicate 5-7 probable SSEs in Hong Kong, and substantial individual heterogeneity in the transmissibility of SARS-CoV-2 infection (Table 1; $k=0.45,95 \%: 0.30-0.72$ ) and therefore potential for future superspreading, albeit less than SARS-CoV and MERS-CoV ( $k=0.16$ and 0.26 respectively) $(15,16)$. Superspreading events pose considerable challenges for local control as they can quickly overwhelm public health contact tracing capacity, and because most infected persons will generate few secondary infections, while a small fraction can generate many (Figure 2B). Indeed, we estimated that approximately $20 \%$ of cases were responsible for $80 \%$ of all SARS-CoV-2 transmission in Hong Kong (Table 1). These results however should be interpreted in the context of constrained community transmission given moderate levels of physical distancing currently practiced in Hong Kong, including school closures, some adults working at home, cancellation of mass gatherings, as well as improved hygiene and universal mask wearing (17). Recent findings from Shenzhen, China estimated comparable levels of overdispersion using contact tracing data $(k=0.58)(18)$, however other studies utilizing global datasets have estimated even greater potential for SARS-CoV-2 superspreading ( $k=0.1$ given a global $\mathrm{R}$ of 2.5 ) and suggest as little as $10 \%$ of cases could account for $80 \%$ of all transmission (19). Such degrees of overdispersion however can be advantageous to disease control efforts if interventions can effectively target the core high-risk group responsible for the majority of transmission $(20,21)$. 
Superspreading is considered a function of both variations in individual transmissibility and individual susceptibility or exposure. Our results show that the number of individual secondary cases was significantly higher within social settings such as bars and restaurants compared to family or work exposures $(\mathrm{p}<0.001)$. This is certainly due to the greater numbers of contacts expected in such settings. Social exposures are therefore at an increased risk for SARS-CoV-2 transmission and likely constitute the core behavioural risk factor for SSEs. Targeted interventions should therefore focus on reducing extreme numbers of social contacts at high-risk venues such as bars, nightclubs and restaurants, which also appear at increased risk of SSE (22), either via closures or physical distancing policies, both of which currently remain implemented in Hong Kong (17). Models have shown however that heterogeneity in exposure may also have disproportionate effects on herd immunity (23), including SARS-CoV-2 $(24,25)$. Because some individuals are at greater risk of exposure, achieving herd immunity in this core risk group may therefore have suppressive effects on local transmission more generally, potentially reducing the otherwise necessary duration and intensity of more disruptive community-wide physical distancing policies. Given a coefficient of variation of 2.5 from our study (Table 1) this roughly corresponds to an adjusted herd immunity threshold of $20 \%$ (25), which is consistent with our estimate that $20 \%$ of individuals are responsible for $80 \%$ of transmission in Hong Kong. This compares to a herd-immunity threshold of $60-70 \%$ via vaccine-acquired immunity. The real-world effects of naturally acquired 
herd immunity however should be further studies and measured with serology along with the progressive easing of restrictions.

Previous modelling has suggested that reduced delays between symptom onset and isolation are important indicators for the control of SARS-CoV-2 outbreaks (20), however this did not appear to play a major role in Hong Kong: in our analysis, delay from symptom onset to isolation were not associated with the number of individual secondary cases, nor the final cluster size (Figure 4A and 4B). In contrast, for SARS-CoV various epidemics in 2003, due to delayed viral shedding among cases (highest approximately seven days after symptom onset) $(26,27)$, delays in case isolation adversely affected disease control efforts (28). For COVID-19, confirmation and isolation of symptomatic cases will have a limited effect on reducing overall SARS-CoV-2 transmission in the community unless done very quickly, noting the growing body of evidence of substantial transmission during the pre-symptomatic and early symptomatic period (29-31). In Hong Kong, the average delay to isolation was 5.6 days for cluster cases (Figure 4), and by this time most onwards transmission had perhaps already occurred. The most important public health measures therefore are likely to be case identification followed by rapid and parallel (e.g. before contacts are confirmed as cases) contact tracing and quarantine. Some degree of intermittent physical distancing may also be required to supress potential local transmission from unidentified infections and pre-symptomatic 
transmission, but must necessarily be balanced with the social, economic and educational costs associated with such policies.

Overall our study has a few limitations. Primarily, because this study relies on the completeness of case ascertainment and contact tracing data, any degree of incompleteness could therefore bias our estimates. Further, because the source of 50 sporadic local infections could not be determined, we likely underestimated $\mathrm{R}$ based on the empirical data alone. In fact, the expected difference between of $\mathrm{R}$ and $k$ from our observed estimates and likelihood model indicates the presence of some incompleteness and therefore potential bias, however only marginally so (Table 1). Secondly, our results also suggest a potential caveat: despite evidence supporting rapid contact tracing and the effectiveness of quarantine (Figure 4), in our analysis, most chains of transmission did not terminate in quarantine (Figure 3;

Supplementary Table 5). This however must be interpreted in the context of moderate physical distancing in Hong Kong as highlighted previously (17), rather than a suggestion that quarantine was not essential.

Overall there is substantial potential for SSEs in COVID-19, but less so than for SARS and MERS. In the absence of non-pharmaceutical interventions such as physical distancing implemented in Hong Kong, the potential for SSEs is likely greater than observed in our study. Assuming local elimination is not possible, disease control efforts should focus on rapid tracing and quarantine of confirmed contacts, along 
with the implementation of physical distancing policies or closures targeting highrisk social exposures such as bars, nightclubs and restaurants to prevent the occurrence of SSEs. Given the long right-hand tail of the distribution of individual reproductive numbers (Figure 2B), preventing SSEs would have a considerable effect in reducing the overall reproductive number. In lieu of an effective vaccine, these results have significant implications for the control of COVID-19 and public health measures such as physical distancing and the relaxation of lockdowns around the world.

\section{METHODS}

Characterisation of clusters and chains of SARS-CoV-2 transmission

Using case line lists and contact tracing data obtained from the Centre for Health Protection (CHP) of the Department of Health in Hong Kong we characterised clusters of SARS-CoV-2 infections and chains of transmission within clusters. Cases of SARS-CoV-2 infection were laboratory confirmed via RT-PCR. Clusters were defined as two or more confirmed infections with close contact prior to or following symptom onset. Each cluster was characterised by the travel history of the index case as either initiated by an imported case (i.e. index acquired SARS-CoV-2 infection overseas), initiated by a local case or clusters of solely imported cases. Cases not linked to any cluster were categorised as sporadic local or sporadic imported cases if infection was acquired locally or overseas. We determined probable infector-infectee transmission pairs and chains of transmission within clusters from reported contact 
histories and symptom onset dates for all clusters initiated by an imported or local case. Transmission pairs were characterised by the reported site of contact as either family, social, work or during local travel such as on public transport and modelled using a negative binomial log-linear model. Transmission between cases within clusters of solely imported cases were not considered due to substantial ambiguities concerning transmission within each cluster whilst overseas.

\section{Calculation of serial interval and empirical offspring distribution}

We calculated the median serial interval as the difference between the symptom onset dates of each infector-infectee pair, excluding asymptomatic cases, and fitted normal, lognormal, gamma and Weibull distributions using the $\mathrm{R}$ package "fitdistrplus" and maximum-likelihood methods, excluding eight non-positive data points for the latter three distributions. We generated the empirical offspring distribution from the observed number of secondary cases per individual infector and similarly fit negative binomial, geometric and Poisson distributions as before. Cases terminal to the inferred chain of transmission and sporadic local cases were considered to have zero secondary cases. We investigated the relationship between delay in days from symptom onset-to-confirmation as a proxy for potential infectiousness in the community and the number of secondary cases per infector by linear regression. We compared each fit distribution using Akaike information criterion (AIC) scores and calculated confidence intervals for parameter from 1000 bootstrapped replicates. 
Superspreading and individual variance of SARS-CoV-2 transmission

Individual variation in SARS-CoV-2 transmission

Following the approach described by Lloyd Smith et al. (15), estimates of the observed reproductive number $(R)$ was determined from the mean of the negative binomial distribution fit to the empirical offspring distribution, and the degree of individual variation on transmission from the corresponding dispersion parameter $k$. We repeated this analysis for both wave one (January-February 2020) and wave two (March-April 2020), sub-setting by illness onset date of the index case. Due to potential biases affecting the empirical offspring distributions, we simultaneously implemented a likelihood-based branching process model to jointly infer $\mathrm{R}$ and $k$ given the final size of all local clusters following Kucharski and Althaus (16) where the probability that an index case generates clusters $n$ of size $j$ is given by $(32,33)$ :

$$
r_{j}=\frac{\Gamma(k j+(j-1)}{\Gamma(k j) \Gamma(j+1)} \frac{\left(\frac{R_{0}}{k}\right)^{j-1}}{\left(1+\frac{R_{0}}{k}\right)^{k j+j-1}}
$$

And the likelihood is:

$$
L=\prod_{j=1}^{\infty} r_{j}^{n_{j}}
$$

For a given range of values for $\mathrm{R}(0.10-3.00)$ and $k(0.01-55)$, the probability of an index case generating a cluster equal to or greater than $j$ is given by: 


$$
p_{j}=1-\sum_{i=1}^{j-1} r_{i}
$$

From the empirical and likelihood model estimates of $\mathrm{R}$ and $k$, we calculated the coefficient of variation as the ratio of standard deviation of the corresponding negative binomial distribution and the mean. Following from Endo et al. (19), given parameters $\mathrm{R}$ and $k$, the expected proportion of cases responsible for $80 \%$ of transmission in Hong Kong is given by:

$$
1-\mathrm{P} 80 \%=\frac{1}{R_{0}} \int_{0}^{X}\lfloor x\rfloor \mathrm{NB}\left(\lfloor x\rfloor ; k, \frac{k}{R_{0}+k}\right) d x
$$

where $\mathrm{X}$ satisfies:

$$
1-0.8=\frac{1}{R_{0}} \int_{0}^{X}\lfloor x\rfloor \mathrm{NB}\left(\lfloor x\rfloor ; k, \frac{k}{R_{0}+k}\right) d x
$$

and:

$$
\frac{1}{R_{0}} \int_{0}^{X}\lfloor x\rfloor \mathrm{NB}\left(\lfloor x\rfloor ; k, \frac{k}{R_{0}+k}\right) d x=\int_{0}^{X-1}\lfloor x\rfloor \mathrm{NB}\left(\lfloor x\rfloor ; k+1, \frac{k}{R_{0}+k}\right) d x
$$

Finally, as per Lloyd Smith et al. (15) given $R_{0}$, the superspreading threshold is calculated as the $99^{\text {th }}$-percentile of the Poisson $\left(R_{0}\right)$ distribution where:

$\operatorname{Pr}\left(Z \leq Z^{(99)} \mid Z \sim \operatorname{Poisson}\left(R_{0}\right)\right)=0.01$. Therefore, with the global consensus of $R_{0}$ in the range $2-3$, we define the superspreading threshold for SARS-CoV-2 here as 6-8 
373 individual secondary cases. All statistical analyses were performed in $\mathrm{R}$ version 4.0.0

374. (R Foundation for Statistical Computing, Vienna, Austria).

375

376 


\section{ACKNOWLEDGMENTS}

This project was supported by a commissioned grant from the Health and Medical Research Fund, Food and Health Bureau, Government of the Hong Kong Special Administrative Region, and the Theme-based Research Scheme [Project No. T11712/19-N] of the Research Grants Council of the Hong Kong SAR Government.

\section{AUTHOR CONTRIBUTIONS}

All authors meet the ICMJE criteria for authorship. The study was conceived by DCA and BJC. Data analyses were done by DCA, JYW and EHYL. DCA wrote the first draft of the manuscript, and all authors provided critical review and revision of the text and approved the final version.

\section{COMPETING INTERESTS STATEMENT}

BJC consults for Roche and Sanofi Pasteur. The authors report no other potential conflicts of interest. 


\section{REFERENCES}

1. World Health Organization. Coronavirus disease 2019 (COVID-19): situation report, 100. 2020.

2. WHO Director-General's opening remarks at the media briefing on COVID-19 11 March 2020 Geneva, Switzerland: World Health Organization; [cited 2020 May 07]. Available from: https://www.who.int/dg/speeches/detail/whodirector-general-s-opening-remarks-at-the-media-briefing-on-covid-19---11march-2020.

3. Wang SX, Li Y, Sun B, Zhang S, Zhao W, Wei M, et al. The SARS outbreak in a general hospital in Tianjin, China-the case of super-spreader. Epidemiology \& Infection. 2006;134(4):786-91.

4. Kim K, Tandi T, Choi JW, Moon J, Kim M. Middle East respiratory syndrome coronavirus (MERS-CoV) outbreak in South Korea, 2015: epidemiology, characteristics and public health implications. Journal of Hospital Infection. 2017;95(2):207-13.

5. Cho SY, Kang J-M, Ha YE, Park GE, Lee JY, Ko J-H, et al. MERS-CoV outbreak following a single patient exposure in an emergency room in South Korea: an epidemiological outbreak study. Lancet. 2016;388(10048):994-1001.

6. Cowling BJ, Park M, Fang VJ, Wu P, Leung GM, Wu JT. Preliminary epidemiological assessment of MERS-CoV outbreak in South Korea, May to June 2015. Euro Surveillance. 2015;20(25):7-13.

7. Galvani AP, May RM. Dimensions of superspreading. Nature. 2005;438(7066):293-5.

8. Wallinga J, Teunis P. Different epidemic curves for severe acute respiratory syndrome reveal similar impacts of control measures. American Journal of Epidemiology, 2004;160(6):509-16.

9. Shen Z, Ning F, Zhou W, He X, Lin C, Chin DP, et al. Superspreading sars events, Beijing, 2003. Emerging infectious diseases. 2004;10(2):256. 
10. Wallinga J, Teunis P. Different epidemic curves for severe acute respiratory syndrome reveal similar impacts of control measures. American Journal of Epidemiology. 2004;160(6):509-16.

11. Cho SY, Kang J-M, Ha YE, Park GE, Lee JY, Ko J-H, et al. MERS-CoV outbreak following a single patient exposure in an emergency room in South Korea: an epidemiological outbreak study. Lancet. 2016;388(10048):994-1001.

12. Zhao S, Lin Q, Ran J, Musa SS, Yang G, Wang W, et al. Preliminary estimation of the basic reproduction number of novel coronavirus (2019-nCoV) in China, from 2019 to 2020: A data-driven analysis in the early phase of the outbreak. International Journal of Infectious Diseases. 2020;92:214-7.

13. Zhang S, Diao M, Yu W, Pei L, Lin Z, Chen D. Estimation of the reproductive number of novel coronavirus (COVID-19) and the probable outbreak size on the Diamond Princess cruise ship: A data-driven analysis. International Journal of Infectious Diseases. 2020;93:201-4.

14. Leung GM, Cowling BJ, Wu JT. From a Sprint to a Marathon in Hong Kong. New England Journal of Medicine. 2020;382(18):e45.

15. Lloyd-Smith JO, Schreiber SJ, Kopp PE, Getz WM. Superspreading and the effect of individual variation on disease emergence. Nature. 2005;438(7066):3559.

16. Kucharski A, Althaus C. The role of superspreading in Middle East respiratory syndrome coronavirus (MERS-CoV) transmission. Euro surveillance. 2015;20(25):pii= 21167.

17. Cowling BJ, Ali ST, Ng TWY, Tsang TK, Li JCM, Fong MW, et al. Impact assessment of non-pharmaceutical interventions against coronavirus disease 2019 and influenza in Hong Kong: an observational study. Lancet Public Health. 2020;5(5):e279-e88.

18. Bi Q, Wu Y, Mei S, Ye C, Zou X, Zhang Z, et al. Epidemiology and transmission of COVID-19 in 391 cases and 1286 of their close contacts in Shenzhen, China: a retrospective cohort study. Lancet Infectious Diseases. 
19. Endo A, null n, Abbott S, Kucharski A, Funk S. Estimating the overdispersion in COVID-19 transmission using outbreak sizes outside China [version 1; peer review: 1 approved]. Wellcome Open Research. 2020;5(67).

20. Hellewell J, Abbott S, Gimma A, Bosse NI, Jarvis CI, Russell TW, et al. Feasibility of controlling COVID-19 outbreaks by isolation of cases and contacts. Lancet Global Health. 2020;8(4):e488-e96.

21. Woolhouse ME, Dye C, Etard J-F, Smith T, Charlwood J, Garnett G, et al. Heterogeneities in the transmission of infectious agents: implications for the design of control programs. Proceedings of the National Academy of Sciences. 1997;94(1):338-42.

22. Lu J, Gu J, Li K, Xu C, Su W, Lai Z, et al. COVID-19 Outbreak Associated with Air Conditioning in Restaurant, Guangzhou, China, 2020. Emerg Infect Dis. $2020 ; 26(7)$.

23. Hill AN, Longini Jr IM. The critical vaccination fraction for heterogeneous epidemic models. Mathematical Biosciences. 2003;181(1):85-106.

24. Britton T, Ball F, Trapman P. The disease-induced herd immunity level for Covid-19 is substantially lower than the classical herd immunity level. arXiv preprint arXiv:200503085. 2020.

25. Gomes MGM, Aguas R, Corder RM, King JG, Langwig KE, Souto-Maior C, et al. Individual variation in susceptibility or exposure to SARS-CoV-2 lowers the herd immunity threshold. medRxiv. 2020.

26. Peiris JS, Chu CM, Cheng VC, Chan KS, Hung IF, Poon LL, et al. Clinical progression and viral load in a community outbreak of coronavirus-associated SARS pneumonia: a prospective study. Lancet. 2003;361(9371):1767-72.

27. Pitzer VE, Leung GM, Lipsitch M. Estimating variability in the transmission of severe acute respiratory syndrome to household contacts in Hong Kong, China. American Journal of Epidemiol. 2007;166(3):355-63.

28. Li Y, Yu ITS, Xu P, Lee JHW, Wong TW, Ooi PL, et al. Predicting Super Spreading Events during the 2003 Severe Acute Respiratory Syndrome 
Epidemics in Hong Kong and Singapore. American Journal of Epidemiology. 2004;160(8):719-28.

29. He X, Lau EH, Wu P, Deng X, Wang J, Hao X, et al. Temporal dynamics in viral shedding and transmissibility of COVID-19. Nature Medicine. 2020:1-4.

30. Ferretti L, Wymant C, Kendall M, Zhao L, Nurtay A, Abeler-Dorner L, et al. Quantifying SARS-CoV-2 transmission suggests epidemic control with digital contact tracing. Science. 2020.

31. Arons MM, Hatfield KM, Reddy SC, Kimball A, James A, Jacobs JR, et al. Presymptomatic SARS-CoV-2 Infections and Transmission in a Skilled Nursing Facility. New England Journal of Medicine. 2020.

32. Blumberg S, Lloyd-Smith JO. Inference of R 0 and transmission heterogeneity from the size distribution of stuttering chains. PLoS Computational Biology. 2013;9(5).

33. Nishiura H, Yan P, Sleeman CK, Mode CJ. Estimating the transmission potential of supercritical processes based on the final size distribution of minor outbreaks. Journal of Theoretical Biology. 2012;294:48-55. 


\section{FIGURE LEGENDS}

Figure 1. Epidemic curve of daily cases of laboratory-confirmed SARS-CoV-2 infection in Hong Kong by symptom onset date and coloured by cluster category $(\mathrm{N}=1,038)$. Important travel and community health interventions are indicated with arrows. Asymptomatic cases are included here by date of confirmation.

Figure 2: (A) Empirical serial distribution of SARS-CoV-2 infections in Hong Kong among symptomatic infector-infectee pairs with fitted normal (solid line) and lognormal (dotted) distributions. The lognormal distribution was fitted excluding observations $\leq 0$ days. (B) Empirical offspring distribution of SARS-CoV-2 among local and imported COVID-19 cases in Hong Kong (excluding clusters of cases where infections had been acquired overseas) and a fitted negative binomial distribution with $\mathrm{R}=0.58$ and $k=0.45$.

Figure 3. Local chains of SARS-CoV-2 transmission in Hong Kong initiated by another local case or an imported case. (A) Transmission network of the "bar and band" cluster of cases. The source case of the initial exposure could not be determined. (B) Transmission network associated with a single wedding exposure subsequently linked to a preceding social gathering and local source exposure. (C) Transmission network associated with a Buddhist temple in Hong Kong. Primary cases report multiple distinct exposures to the temple over the course of many days. It is suspected that an asymptomatic monk at the temple may have been the source 
(indicated by the pink circle) however it cannot be known if they were exposed to another case, possibly an environmental exposure. (D) All other clusters of SARSCoV-2 infections initiated by local and imported cases where the source and transmission chain could be determined. Footnote 1: An asymptomatic employee at one of the bars was placed in quarantine on 26 March following recognition of the cluster and tested positive on 4 April. Was transferred to hospital for observation but did not develop symptoms. This employee's wife developed symptoms on 1 April and was confirmed to have SARS-CoV-2 infection on 6 April.

Figure 4. (A) Distribution of delays in days from symptom onset to isolation of cases by cluster size. (B) Delays from symptom onset to isolation of index cases by the number of secondary cases per index coloured by site of transmission (Dark Blue: Family, Red: Social, Green: Local travel, Light Blue: Work) 
534 Table 1: Estimated effective reproductive number, overdispersion parameter,

535 coefficient of variation and proportion of infectious cases responsible for $80 \%$ of

536 transmission.

\begin{tabular}{|c|c|c|c|c|}
\hline Distribution/Model & R $(95 \%$ CI $)$ & k $(95 \% \mathrm{CI})$ & $\begin{array}{r}\text { Coefficient of } \\
\text { variation }(95 \% \mathrm{CI})\end{array}$ & $\begin{array}{r}\text { Prop80\%* } \\
(95 \% \text { CI })\end{array}$ \\
\hline Empirical Offspring & $0.58(0.45-0.71)$ & $0.45(0.30-0.72)$ & $2.5(1.8-3.3)$ & $20 \%(15 \%-25 \%)$ \\
\hline Branching Process & $0.74(0.58-0.96)$ & $0.33(0.15-0.97)$ & $2.6(1.4-4.3)$ & $17 \%(11 \%-29 \%)$ \\
\hline
\end{tabular}

537 *Prop $80 \%$ is the proportion of cases responsible for $80 \%$ of transmission 
Figures

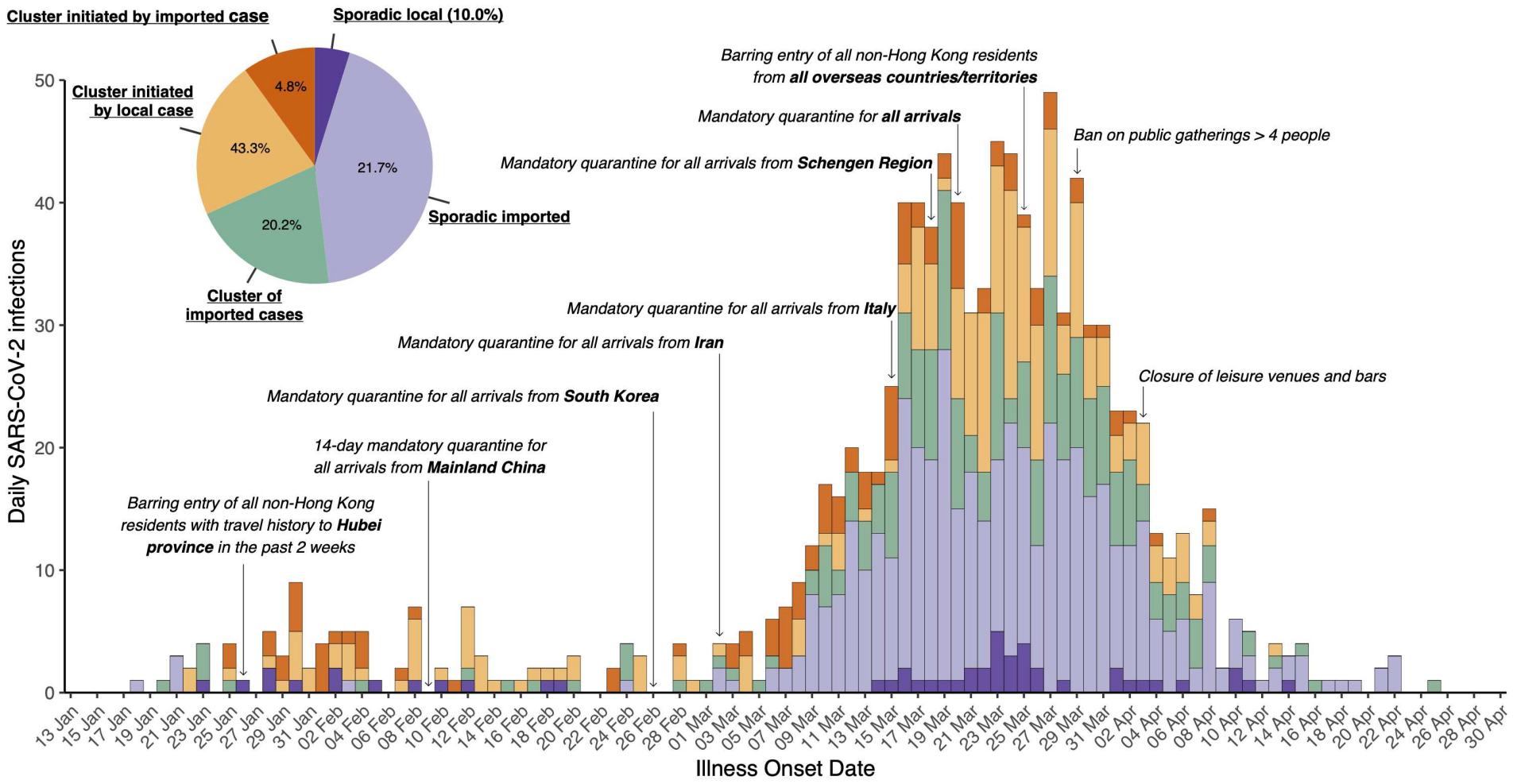

\section{Figure 1}

Epidemic curve of daily cases of laboratory-confirmed SARS-CoV-2 infection in Hong Kong by symptom onset date and coloured by cluster category $(\mathrm{N}=1,038)$. Important travel and community health interventions are indicated with arrows. Asymptomatic cases are included here by date of confirmation.
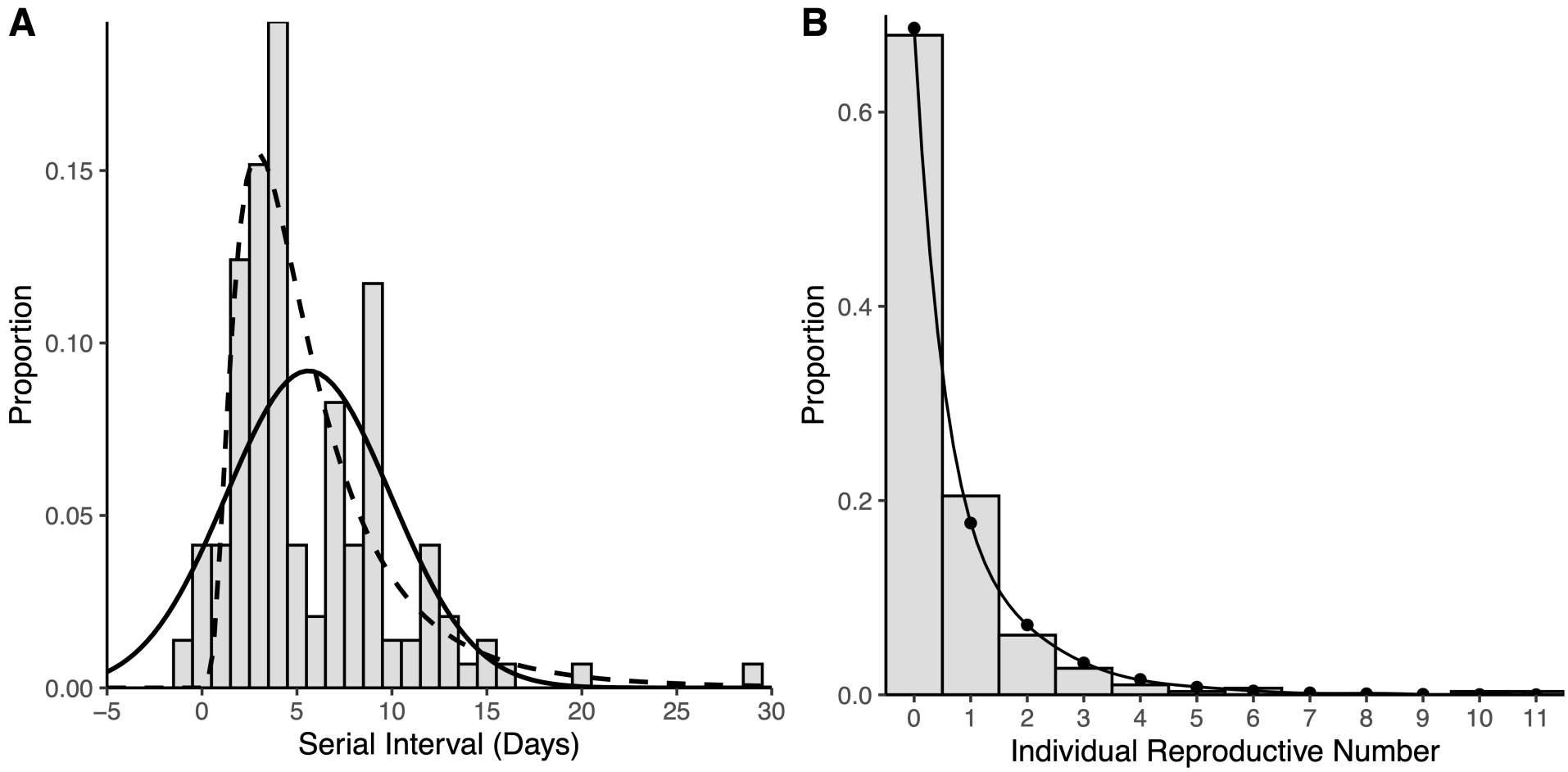
Figure 2

(A) Empirical serial distribution of SARS-CoV-2 infections in Hong Kong among symptomatic infectorinfectee pairs with fitted normal (solid line) and lognormal (dotted) distributions. The lognormal distribution was fitted excluding observations $\leq 0$ days. (B) Empirical offspring distribution of SARS-CoV-2 among local and imported COVID-19 cases in Hong Kong (excluding clusters of cases where infections had been acquired overseas) and a fitted negative binomial distribution with $\mathrm{R}=0.58$ and $\mathrm{k}=0.45$.
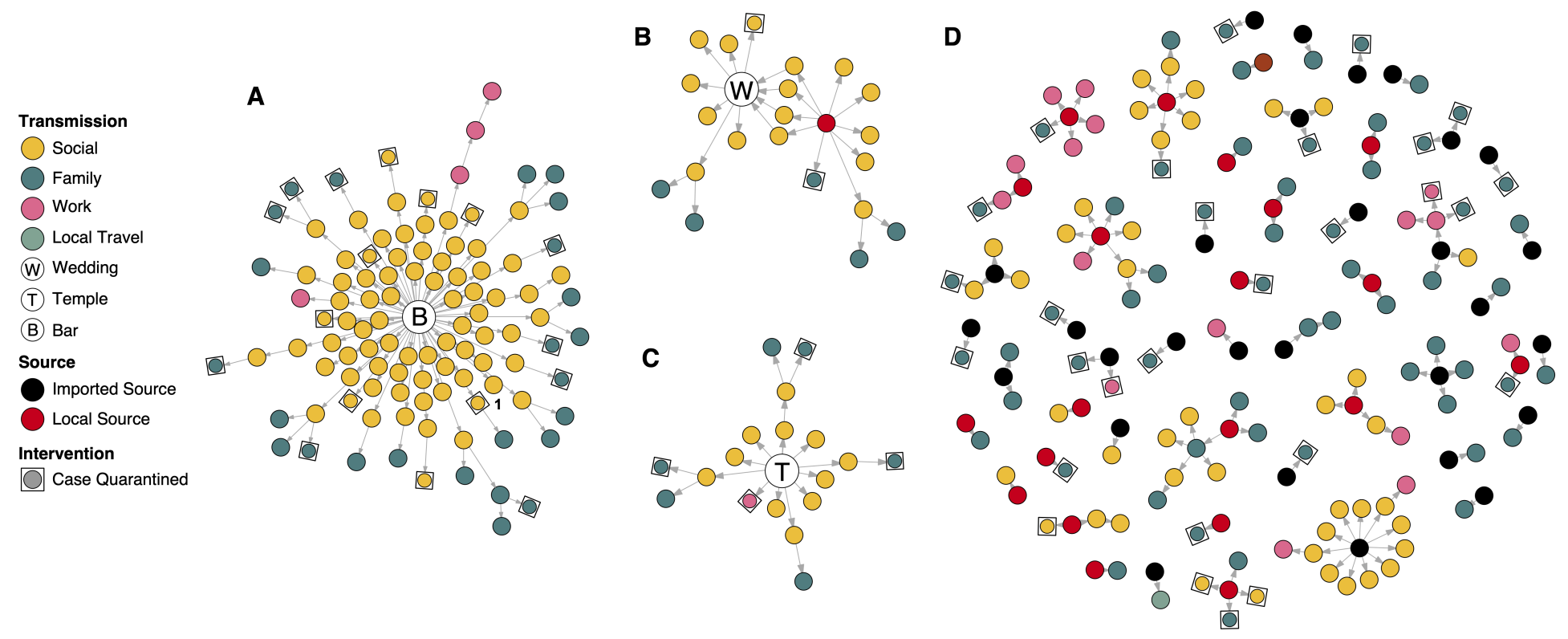

\section{Figure 3}

Local chains of SARS-CoV-2 transmission in Hong Kong initiated by another local case or an imported case. (A) Transmission network of the "bar and band" cluster of cases. The source case of the initial exposure could not be determined. (B) Transmission network associated with a single wedding exposure subsequently linked to a preceding social gathering and local source exposure. (C) Transmission network associated with a Buddhist temple in Hong Kong. Primary cases report multiple distinct exposures to the temple over the course of many days. It is suspected that an asymptomatic monk at the temple may have been the source (indicated by the pink circle) however it cannot be known if they were exposed to another case, possibly an environmental exposure. (D) All other clusters of SARS-CoV-2 infections initiated by local and imported cases where the source and transmission chain could be determined. Footnote 1: An asymptomatic employee at one of the bars was placed in quarantine on 26 March following recognition of the cluster and tested positive on 4 April. Was transferred to hospital for observation but did not develop symptoms. This employee's wife developed symptoms on 1 April and was confirmed to have SARS-CoV-2 infection on 6 April. 

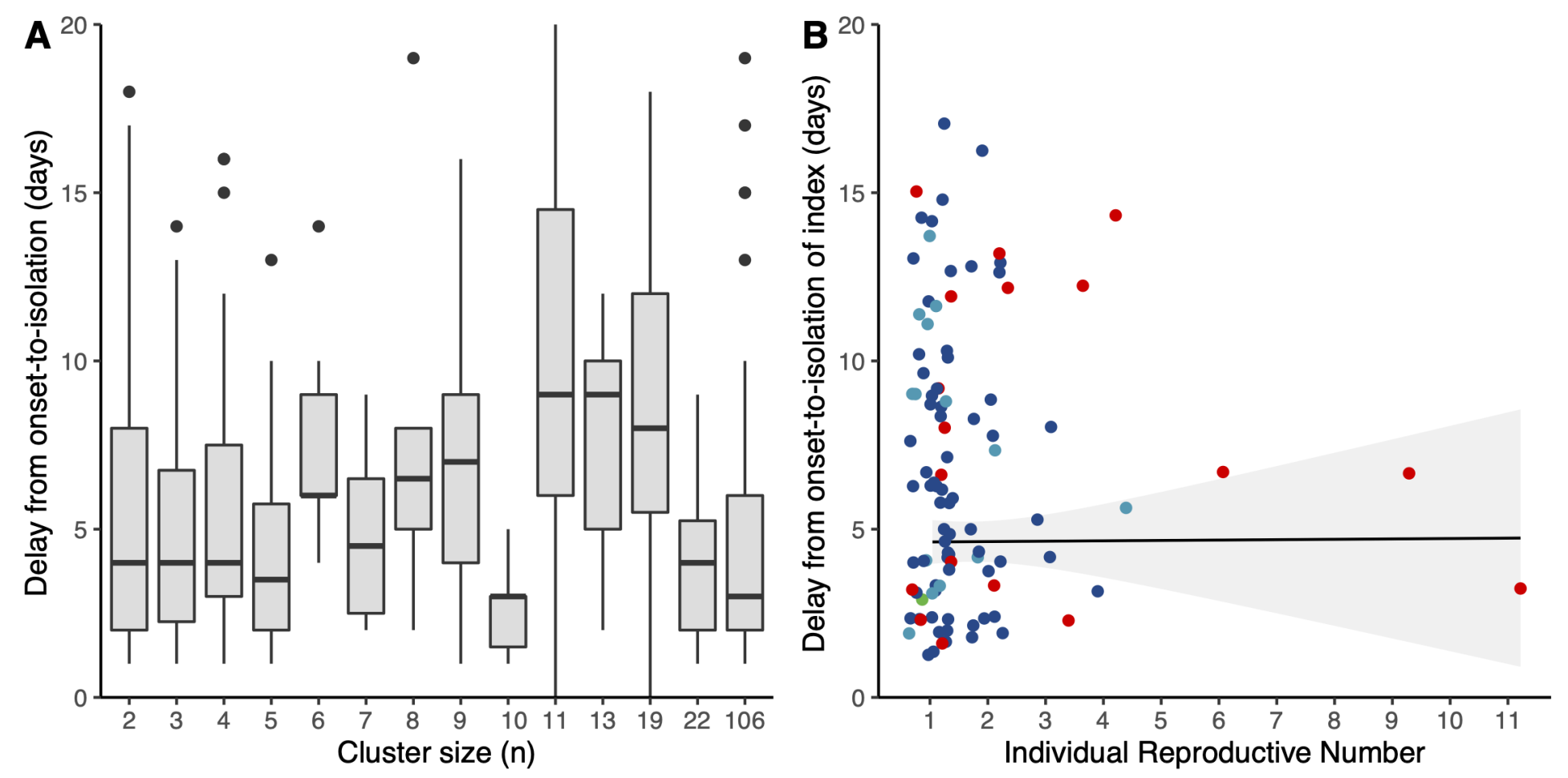

Figure 4

(A) Distribution of delays in days from symptom onset to isolation of cases by cluster size. (B) Delays from symptom onset to isolation of index cases by the number of secondary cases per index coloured by site of transmission (Dark Blue: Family, Red: Social, Green: Local travel, Light Blue: Work)

\section{Supplementary Files}

This is a list of supplementary files associated with this preprint. Click to download.

- covidssesupplementary.v18.May.20.docx 